\title{
Visualization of Gold Nanoparticle Self-assembly Kinetics
}

Taylor J. Woehl ${ }^{1}$ and Tanya Prozorov ${ }^{1}$

1. Emergent Atomic and Magnetic Structures, Division of Materials Sciences and Engineering, Ames Laboratory, Ames, IA 50011, USA.

Various types of colloidal nanoparticles are known to self-assemble into hierarchical mesostructures via anisotropic interparticle interactions, such as dipolar [1] and electrostatic interactions [2]. Self-assembly strategies are typically designed in an empirical manner or based on equilibrium models of these interparticle interactions, and kinetic considerations are often overlooked. Due to a lack of high spatial and temporal resolution in situ observations, little is known about the kinetics of nanoparticle selfassembly. Previous work has suggested that the kinetics of nanoparticle self-assembly process can be likened to multistep chemical [3] and polymerization [4] reactions, where interparticle interactions determine the rate constants for assembly of differently sized and shaped mesostructures [5,6]. However, it is not clear how the interplay of interparticle interactions and assembly kinetics affect the hierarchical self-assembly process and resulting mesostructure morphology.

Here we use real-time nanoscale observations to measure the self-assembly kinetics of colloidal gold nanoparticles into one dimensional chains. Gold nanoparticles suspended in acetate buffer were observed with in situ liquid cell scanning transmission electron microscopy (STEM) to self-assemble into chains of up to 20 nanoparticles over times of several minutes. Real-time kinetic data and in situ nanometer resolution images revealed the formation pathway and rate of gold nanoparticle self-assembly to be dependent on the imaging electron beam current (Figure 1). The rate of self-assembly increased proportionally with the beam current, and the self-assembly pathway changed from sequential attachment of nanoparticles to formed chains at low beam currents, to chain-chain attachments at high beam currents. Experimental measurements of the nanoparticle diffusion coefficient revealed that the self-assembly process was diffusion driven, where the nanoparticle mobility dictated the self-assembly rate and pathway. Importantly, through these systematic beam current experiments we revealed that the nanoparticle mobility was the underlying control factor for the self-assembly kinetics. We expect these conclusions will shed light on the role of other mobility-controlling factors on nanoparticle selfassembly, such as nanoparticle size, shape, and suspending solvent [7].

\section{References:}

[1] Z.Y. Tang, N.A. Kotov, M. Giersig, Science, 297 (2002), p. 237-240.

[2] H. Zhang, D.Y. Wang, Angewandte Chemie-International Edition, 47 (2008), p. 3984.

[3] Q. Zhou et al, Crystengcomm, 15 (2013), p. 5114.

[4] K. Liu et al, Science, 329 (2010), p. 197.

[5] J. Zhang et al, Journal of the American Chemical Society, 128 (2006), p. 12981.

[6] R.L. Penn, Journal of Physical Chemistry B, 108 (2004), p. 12707.

[7] We thank Cari Dutch and William Ristenpart for assistance in developing the image analysis algorithms for single particle tracking and diffusivity measurements. T.P. acknowledges support from the Department of Energy Office of Science Early Career Research Award, Biomolecular Materials Program. This work was supported by the U.S. Department of Energy, Office of Basic Energy Science, Division of Materials Sciences and Engineering. The research was performed at the Ames Laboratory, 
which is operated for the U.S. Department of Energy by Iowa State University under Contract No. DEAC02-07CH11358.


Figure 1. Electron beam dependent gold nanoparticle self-assembly kinetics. Time lapsed annular dark field (ADF) STEM images showing self-assembly of gold nanoparticle chains over $150 \mathrm{~s}$ at beam currents of (a) $107 p A$ and (b) $634 p A$. The scale bar in the final panel of (b) is $200 \mathrm{~nm}$. (c)-(d) The number of nanoparticle chains with sizes ranging from $1-5$ nanoparticles, as a function of time for beam currents of (c) $107 p A$ and (d) $634 p A$. 